\title{
ARTICLE
}

\section{Preliminary shielding design of CSNS day-one neutron instruments}

\author{
Fei Shen ${ }^{\mathrm{a}, \mathrm{b}, \mathrm{c}}$, Tai-ran Liang ${ }^{\mathrm{b}, \mathrm{c}, \mathrm{d}}$, Wan-ju Luo ${ }^{\mathrm{c}}$, Wen Yin ${ }^{\mathrm{b}}$, \\ Quan-zhi $\mathrm{Yu}^{\mathrm{b}}$, Zhi-rong Zeng ${ }^{\mathrm{c}}$ and Tian-jiao Liang ${ }^{\mathrm{b}^{*}}$ \\ ${ }^{a}$ The School of Nuclear Science and Technology, Lanzhou University, Lanzhou 730000, China; ${ }^{b}$ Institute of Physics, Chinese \\ Academy of Sciences, Beijing 100190, China; ${ }^{c}$ Institute of High Energy Physics, Chinese Academy of Sciences, Beijing \\ 100049, China; ${ }^{d}$ Department of Physics, Nankai University, Tianjin 300071, China
}

\begin{abstract}
The China Spallation Neutron Source (CSNS), intended to operate in 2018, is designed with $1.6 \mathrm{GeV}$ proton energy and $25 \mathrm{~Hz}$ repetition rates, with an initial proton beam power of $100 \mathrm{~kW}$ that can be upgradable to $500 \mathrm{~kW}$. Twenty neutron beam lines are provided by the CSNS target station for neutron scattering and neutron science research. The three neutron scattering instruments to be operated on day-one are the general purpose powder diffractometer, the multi-purpose reflectometer and the small angle neutron scattering instrument. These instruments present dissimilar shielding issues and their shielding calculation methods are presented in this paper. The thermal, fast and high-energy neutron source terms with angular distribution are individually calculated using target-moderator-reflector geometry and surface tally at a $1 \mathrm{~m}$ distance to the moderator. The thermal neutron losses in the guide system are calculated using a ray-tracing simulation. Possible worse case scenarios are also considered in the shielding analyses. Variance reduction methods, such as source biasing, are employed to speed up the convergence of calculations. As an example, multi-purpose reflectometer shielding analysis is introduced in this paper. All calculations are normalized to $500 \mathrm{~kW}$ proton beam power and a $2.5 \mu \mathrm{Sv} / \mathrm{h}$ dose limitation are applied in accessible locations.
\end{abstract}

Keywords: Monte Carlo; shielding design; neutron scattering instrument; variance reduction; CSNS; source term; the multi-purpose reflectometer

\section{Introduction}

The China Spallation Neutron Source (CSNS), intended to operate in 2018, has started construction in Dongguan, Guangdong, China. The CSNS target station will take $100 \mathrm{~kW}, 1.6 \mathrm{GeV}$, and $25 \mathrm{~Hz}$ proton beam from the accelerator at phase one, and provide 20 neutron beam lines for the neutron scattering and neutron science instruments [1-2]. However, only three neutron scattering instruments, namely, general purpose powder diffractometer (GPPD), multi-purpose reflectometer (MR), and small angle neutron scattering (SANS) instruments, will be constructed on the first day due to a limited budget.

The neutrons emitted from the target-moderatorreflector (TMR) area in the target station include a large fraction of fast and high-energy neutrons with energies up to the incident proton energy, challenging the biological shielding design of the instruments with regard to guaranteeing access with the dose rate during normal operation [3]. The fast and high-energy neutrons with extreme penetration also give rise to the background of the instruments and the need to be suppressed by employing choppers or curved neutron

*Corresponding author. Email: tjliang@aphy.iphy.ac.cn guides.

In the future, the CSNS plans to upgrade the proton beam power to $500 \mathrm{~kW}$. Therefore, the shielding analyses for all day-one instruments are performed according to this beam power. The dose rate limitation for accessible locations is $2.5 \mu \mathrm{Sv} / \mathrm{h}$.

These day-one instruments require specific shielding design considerations because of the diverse beamline length, moderator location and type, sample scattering chamber, different geometry of neutron optics [curve guide (MR), straight guide (SANS), and absence of guide (GPPD)], and presence or absence of T0 chopper, bandwidth choppers, and second shutter. The comparisons of the three instruments for day-one from the viewpoint of the shielding design are summarized in Table 1. The Monte Carlo shielding analyses for the MR, designed for probing magnetic and biological thin films with nanometer scale structure, are presented to demonstrate the calculation methods and results.

\section{Methodology}

The shielding calculation of SANS and GPPD were performed by FLUKA [4], whereas that of MR was by MCNPX 2.5.0 [5]. The neutron and gamma dose maps 
were tallied using mesh-tallies. The variance reduction technologies applied to improve the efficiency of the calculations are listed as follows:

1. Geometry splitting and Russian roulette, to push the particle population radially outward with respect to the beam centerline;

2. Source angle bias, to increase the sampling of near the normal direction of the moderator and enhance the particle number that reaches the far end of the beamline;

3. Source energy bias, to increase the sampling of fast and high-energy neutrons that contribute more to the dose outside of the shielding than the thermal neutron;

4. Source position bias, to increase the sampling of neutrons that enter the guide of the beamline with a small cross-section;

5. Multistep calculations for the TMR area, the neutron transport area of instruments, and the scattering room area of instruments, to speed up the convergence of calculation.

Several possible worse case scenarios were considered in the shielding analyses, such as choppers or second shutter close, for calculating the neutron transport area of instruments, choppers open and thick sample in the sample position or special reflect/ scattering direction for scattering room analyses, choppers open and without sample for beam dump analyses. Then the maximum requirement of shielding is chosen for engineering design.

The geometry and results of SANS neutron transport area shielding calculation with and without the source angle and energy bias are presented in Figure 1. Two calculations employed the same geometry and material, but have different calculation time: $70 \mathrm{~h}$ for the no-biasing source case and $11 \mathrm{~h}$ for the biasing source case. (2.50 GHz, Intel(R) Core(TM) 2 Quad CPU Q8300, four-core parallel computing mode.) A better statistics was obtained in the biasing source case.

Table1. Comparisons of three day-one instruments from the viewpoint of the shielding design.

\begin{tabular}{|l|c|c|c|}
\hline Moderator & $\begin{array}{c}\text { MR } \\
\text { Hydrogen } \\
(20 \mathrm{~K})\end{array}$ & $\begin{array}{c}\text { SANS } \\
\text { Hydrogen } \\
(20 \mathrm{~K})\end{array}$ & $\begin{array}{c}\text { GPPD } \\
\text { \& Poisoned } \\
\text { Hydrogen } \\
(20 \mathrm{~K})\end{array}$ \\
\hline $\begin{array}{l}\text { Neutron } \\
\text { Guide }\end{array}$ & $\begin{array}{c}\text { Curve guide } \\
\text { Taper guide }\end{array}$ & No & $\begin{array}{c}\text { Straight } \\
\text { guide Taper } \\
\text { guide }\end{array}$ \\
\hline $\begin{array}{l}\text { T0 chopper } \\
\text { shutter }\end{array}$ & No & Yes & Yes \\
\hline $\begin{array}{l}\text { Beamline } \\
\text { length }\end{array}$ & $\begin{array}{c}21.5 \mathrm{~m} \text { (to } \\
\text { detector) }\end{array}$ & $\begin{array}{c}16 \mathrm{~m} \text { (to } \\
\text { detector) }\end{array}$ & $\begin{array}{c}\text { No m (to } \\
\text { sample) }\end{array}$ \\
\hline $\begin{array}{l}\text { Collimator } \\
\text { /slit }\end{array}$ & $\begin{array}{c}\text { Slit (size } \\
\text { changeable) }\end{array}$ & $\begin{array}{c}\text { Collimator } \\
+ \text { slits }\end{array}$ & $\begin{array}{c}\text { Collimator } \\
\text { (size } \\
\text { changeable) }\end{array}$ \\
\hline
\end{tabular}
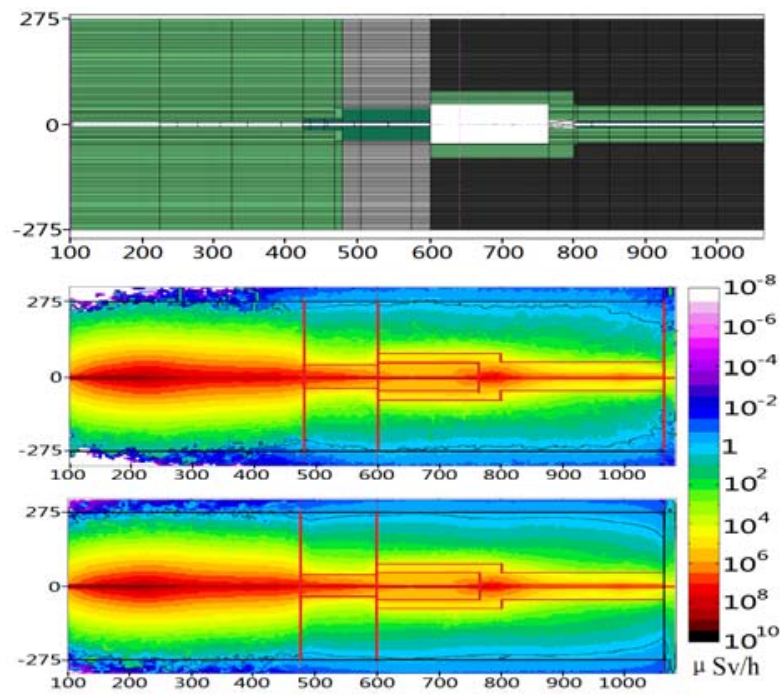

Figure 1. Geometry of SANS neutron transport area (up) and total dose rate (neutron and gamma) for the case without source bias (middle) and with the source angle and energy bias (down). The dimensions of the axes are $\mathrm{cm}$. The color marks in geometry: gray-high density concrete, black-regular concrete, light green-low carbon steel, dark green-SS304.

\section{Source terms}

The neutron source terms from the TMR areas were obtained by a separated calculation with the TMR geometry model and tallied at the core vessel opening of the forward beamlines (41 degree to proton direction) for conservative estimation of source term. Two or three angular groups were tallied within cones of $0-0.5,0.5-1$, and 1-2 degrees, with regard to the normal direction of the moderators. The results are shown in Figure 2.

Besides the TMR source term, the thermal and cold neutron losses of the guides are other source terms that some locations of the instruments. The dedicated neutron ray-tracing code, VITESS [6] or McStas [7], was applied to calculate the neutron beam loss rate along the neutron guides, because both the MCNPX 2.5.0 and

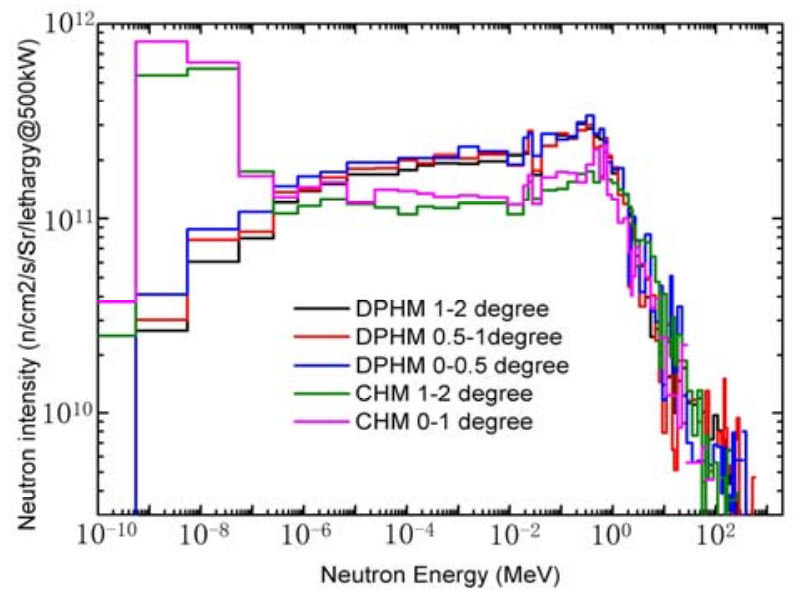

Figure 2. Neutron lethargy spectra of the forward beamline view the coupled hydrogen moderator (CHM) and decoupled and poisoned hydrogen moderator (DPHM), tallied at the core vessel opening at $1-\mathrm{m}$ to moderator. 


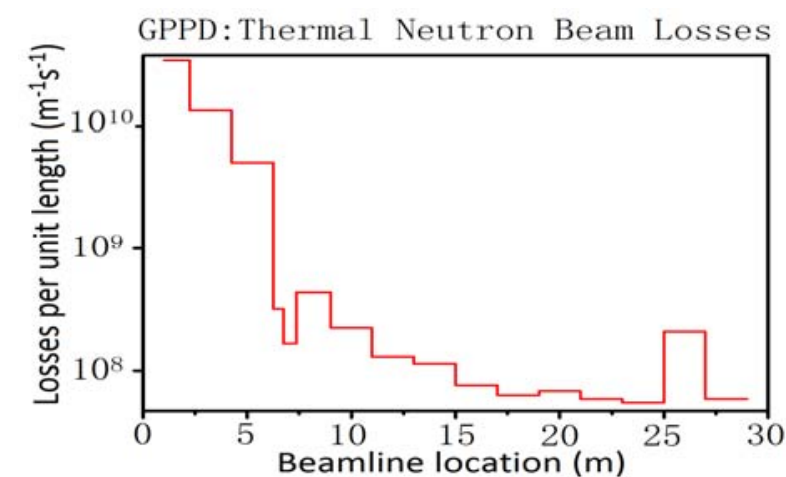

Figure 3. Cold and thermal neutron losses of the GPPD guide system normalized to $500 \mathrm{~kW}$.
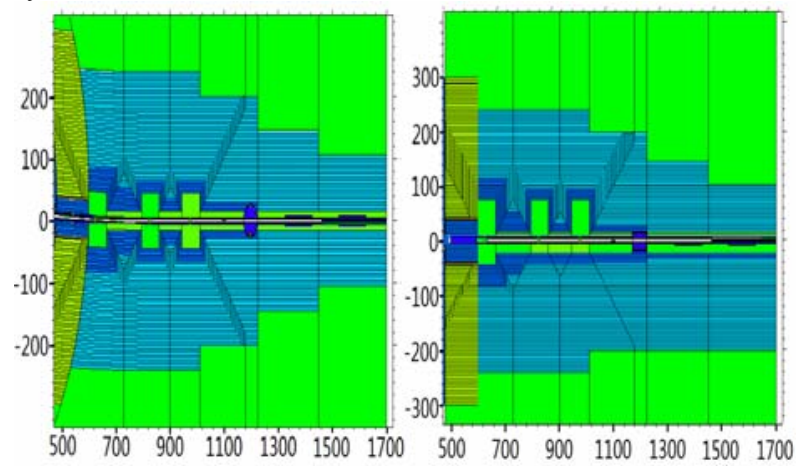

Figure 4. Horizontal (left) and vertical (right) views of the MR beamline shielding geometry. The dimensions of the axes $(\mathrm{cm})$, the color marks: dark blue-low carbon steel, green-air, light blue-regular concrete, and yellow-high density concrete.
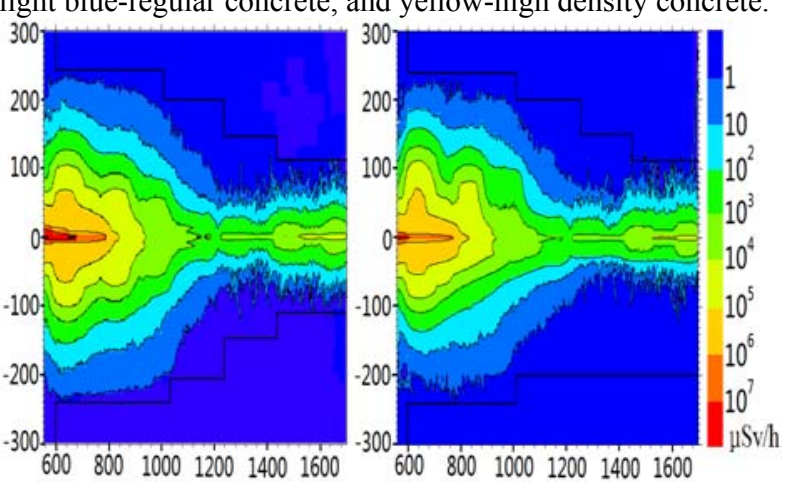

Figure 5. The horizontal (left) and vertical (right) views of total dose rate (neutron and gamma) contour map of MR beamline.

the FLUKA code lacked the ability to simulate the cold and thermal neutron reflection in the super mirror guides. As an example, Figure 3 shows the cold and thermal neutron loss rate versus the beamline location of GPPD. The new neutron source file for the FLUKA simulations and the SDEF card for MCNPX simulations were generated according to the source terms described previously.

\section{Results of the MR shielding calculation}

The horizontal and vertical views of the MR beamline shielding calculation geometry are shown in Figure 4. Both the shielding height and width varies with the beamline length, with regard to the beamline center. In addition, both the concrete outer boundary of height and width are $240 \mathrm{~cm}$, extending from the length to moderator of $6 \mathrm{~m}$ to $10.1 \mathrm{~m}, 200 \mathrm{~cm}$ from the length of $10.1 \mathrm{~m}$ to $12.5 \mathrm{~m}, 150 \mathrm{~cm}$ from the length of $12.5 \mathrm{~m}$ to $14.5 \mathrm{~m}$, and $110 \mathrm{~cm}$ from the length of $14.5 \mathrm{~m}$ to $17 \mathrm{~m}$. The horizontal and vertical views of the dose contour maps are presented in Figure 5. The contour maps indicate that the total dose (neutron and gamma dose) at the outer shielding side is below the dose limitation.

The horizontal and vertical views of the scattering room geometry of MR are shown in Figure 6. Neither the detector bank nor the flippers and polalizers were included in this model. The sample position is located at $2.5 \mathrm{~m}$ downstream of the neutron guide exit. The beam stop, at a position of $6.5 \mathrm{~m}$ downstream of the sample position and connected to a get-lost tube that start at $2.5 \mathrm{~m}$ distance from sample position, was also included in the model. The beam stop consists of a hollow low carbon steel cylinder with $250 \mathrm{~mm}$ inner diameter and $310 \mathrm{~mm}$ outer diameter as well as a base plate with150-mm thickness steel covered on the inside with a 2-mm thick boron carbide layer. The steel cylinder is enclosed in a 200-mm thick sleeve of regular concrete. The un-chopped (choppers fixed at open position) cold and thermal neutron beams at the guide exit through three slits with a maximum opening of $40 \mathrm{~mm} \times 40 \mathrm{~mm}$

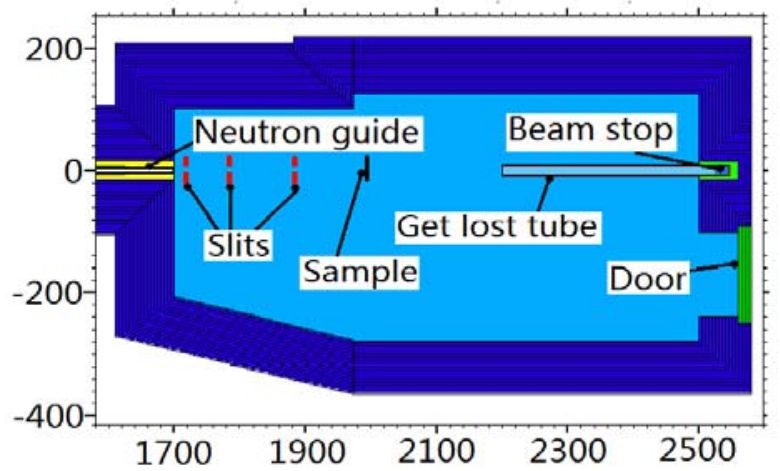

Figure 6. Horizontal view of the geometry of MR scattering room. The dimensions of the axes are $\mathrm{cm}$. The color marks: green-low carbon steel, dark blue-regular concrete, light blue-air, yellow-iron sand, and red-boron carbide. Iron sand means small iron balls enclosed into bags, its density is $2.6 \mathrm{~g} / \mathrm{cc}$ for conservation.

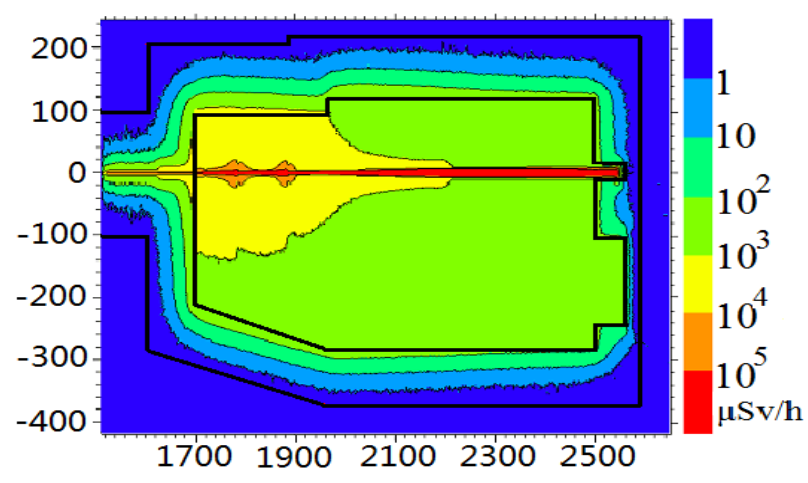

Figure 7. Horizontal view of the total dose rate (neutron and gamma) contour map of the MR scattering room for the case of maximal slits opening and without sample. 
are considered as worse case source terms for the beam stop calculation. The horizontal view of the dose contour maps is shown in Figure 7.

Considering the scattering effects of the samples, the calculations that applied the worse case source terms on worse case samples (10-mm thick water and manganese samples) were performed. Water was chosen because of its high thermal neutron scattering ability; manganese was chosen because of the high-energy gamma ray (up to $8 \mathrm{MeV}$ ) emission during the radiation capture of the thermal neutron. The total dose rate outside the scattering room (Figure 8) is below the limitation. The comparison of the total dose rate (Figure 8) and neutron dose rate (Figure 9) also indicate the difference of the neutron and gamma dose rate distributions due to the choosing of different samples.

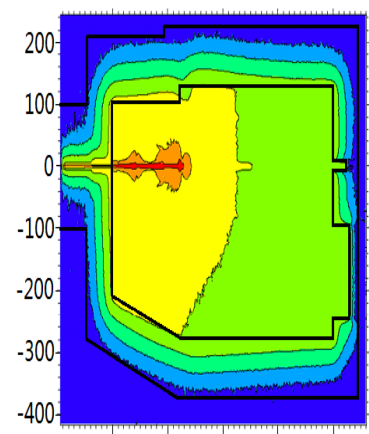

17001900210023002500

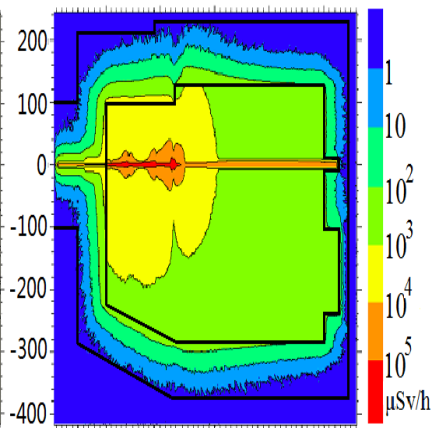

17001900210023002500
Figure 8. Horizontal views of the total dose rate (neutron and gamma) contour map of the MR scattering room caused by the maximal thermal neutron beam incident on a $1-\mathrm{cm}$ thick water sample (left) and a 1-cm thick manganese sample (right).

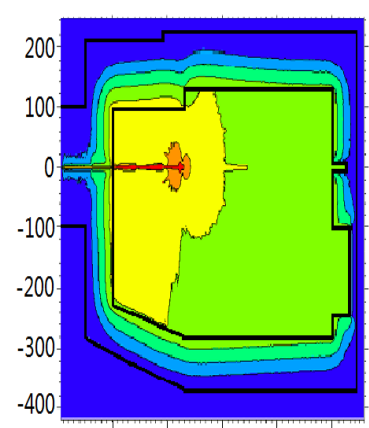

17001900210023002500

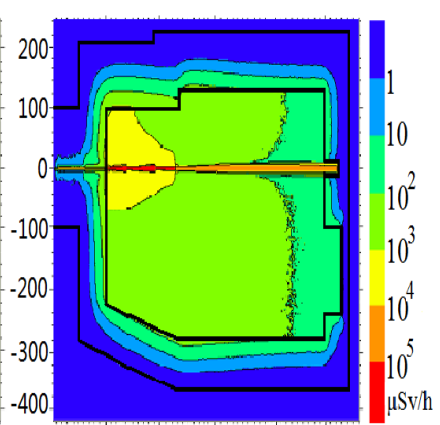

17001900210023002500
Figure 9. Horizontal views of the neutron dose rate contour map of the MR scattering room caused by the maximal thermal neutron beam incident on one a $1-\mathrm{cm}$ thick water sample (left) and a $1-\mathrm{cm}$ thick manganese sample (right).

\section{Conclusion}

The shielding calculations of CSNS day-one instruments were performed to generate the shielding parameters for engineering design instruments. Particularly, the case of MR was discussed in detail including the transport areas and the scattering room in this paper. Variance reduction methods were employed to speed up the convergence of the calculation. Worse case scenarios were also considered for the different locations of the instruments. A dose limitation of $2.5 \mu \mathrm{Sv} / \mathrm{h}$ was achieved for most parts of the instruments shielding.

\section{Acknowledgements}

The authors would like to thank Tai-Seng Zuo, Tao Zhu of MR, Liang Zhou, and Ju-zhou Tao of SANS, Lun-hua He, Jie Chen, and Le Kang of GPPD, and Franz X. Gallmeier, Irina Popova, Phillip D. Ferguson, and Wei Lu of the SNS Neutronics Group for the useful discussion of instrument design and the calculation methods of the instruments shielding. This work was supported by the National Science Foundation of China (Grant No. 91026009 11174358, and 11075203).

\section{References}

[1] W. Yin, T.J. Liang and Q.Z. Yu, Neutronics design for the coupled para-hydrogen moderator for CSNS, Nuclear Instruments and Methods in Physics Research A 631 (2011), pp. 105-110.

[2] J. Wei, H.S. Chen, Y.W. Chen et al., China spallation neutron source: design, R\&D, and outlook, Nuclear Instruments and Methods in Physics Research A 600 (2009), pp. 10-13.

[3] F. Gallmeier, P. Ferguson, E. Iverson, I. Popova and W. Lu, Neutron beamline shielding calculations at the SNS, Nuclear Instruments and Methods in Physics Research A 562 (2006), pp. 946-949.

[4] A. Ferrari, P. R. Sala, A. Fasso and J. Ranft, FLUKA: A Multiparticle Transport Code, CERN-2005-10, INFN0TC-05011, SLAC-R-773, CERN-2005, (2005).

[5] MCNP/MCNPX CCC-730 Monte Carlo N-Particle Transport Code System Including MCNP5.1.40 and MCNPX2.5.0 and Data Libraries, (2005).

[6] G. Zsigmond, K. Lieutenant and F. Mezei, Monte Carlo simulation of neutron scattering instruments by VITESS: Virtual Instrumentation Tool for ESS, Neutron News 13 (2002), pp. 11-14.

[7] K. Lefmann and K. Nielsen, McStas, a general software package for neutron ray-tracing simulations, Neutron News 10 (1999), pp. 20-23. 